\title{
Visual Interpretation of Brain Hypometabolisms Related to Neurological Long COVID: A French Multicentric Experience
}

\section{Antoine Verger}

CHRU de Nancy: Centre hospitalier regional universitaire de Nancy

\section{Aurélie Kas}

APHP: Assistance Publique - Hopitaux de Paris

\section{Pierre Dudouet}

AP-HM: Assistance Publique Hopitaux de Marseille

\section{François Goehringer}

CHRU de Nancy: Centre hospitalier regional universitaire de Nancy

\section{Dominique Salmon}

APHP: Assistance Publique - Hopitaux de Paris

Eric Guedj ( $\nabla$ eric.guedj@ap-hm.fr)

CHU Timone https://orcid.org/0000-0003-1912-6132

\section{Short Report}

Keywords: SARS-CoV-2, COVID-19, long COVID, FDG, PET, olfactory bulb, limbic regions, brainstem, cerebellum

Posted Date: December 20th, 2021

DOI: https://doi.org/10.21203/rs.3.rs-1159513/v1

License: (9) This work is licensed under a Creative Commons Attribution 4.0 International License. Read Full License 


\section{Abstract}

This multicenter study aims to provide a qualitative and consensual description of brain ${ }^{18} \mathrm{~F}$-FDG PET images in patients with suspected neurological long COVID, regarding the previously reported pattern involving olfactory bulbs and other limbic/paralimbic regions, as well as the brainstem and cerebellum.

Methods: From the beginning of August 2021 to the end of October 2021, brain ${ }^{18} \mathrm{~F}-\mathrm{FDG}$ PET exams of patients referred for suspected neurological long COVID with positive reverse transcription polymerase chain reaction (RT-PCR) and/or serology for the SARS-CoV-2 infection were retrospectively reviewed in three French Nuclear Medicine departments. Experimented nuclear physicians from each department had to classify according to the same visual interpretation analysis brain ${ }^{18} \mathrm{~F}$-FDG PET scans as being normal, mildly to moderate (incomplete or moderately hypometabolic), or severely affected within the previously reported long COVID hypometabolic pattern.

Results: On the 143 brain ${ }^{18}$ F-FDG PET performed during this period 3 months, $53 \%$ of scans were visually interpreted as normal, $31 \%$ as mildly to moderate and $16 \%$ as severely affected according to the COVID hypometabolic pattern. Importantly, this specific hypometabolic pattern is reported as identical in the three Nuclear Medicine departments. Typical illustrative examples are provided to help nuclear physicians in the interpretation of long COVID pattern.

Conclusion: The proposed PET metabolic pattern is easily identified at visual interpretation in clinical routine in part of patients with suspicion of neurological long COVID, requiring special consideration for fronto-basal paramedian regions, the brainstem and cerebellum.

\section{Introduction}

Long COVID, defined by the persistence or recurrence of symptoms three months after initial severe acute respiratory syndrome coronavirus 2 (SARS-CoV-2) infection, represents a huge public issue since it approximately affects $10-15 \%$ of patients, with emerging evidence of brain impairment [1-3]. In case of neurocognitive symptoms, these patients may be addressed in nuclear medicine departments for brain ${ }^{18}$ F-FDG PET to contribute to the workup of differential diagnosis, and to objective the hypometabolic pattern previously reported at group-level for this new entity on a recent monocentric study, involving fronto-orbital olfactory regions and other limbic/paralimbic regions, as well as the brainstem and cerebellum [4]. Brain areas which composed this hypometabolic pattern have also been reported in an MRI study measuring brain atrophy before and after the COVID infection [2], as well as in a review of ninety imaging studies [3]. Validation of this long COVID hypometabolic PET pattern is consequently needed at individual- and multicentric-levels to easily transpose this biomarker in clinical routine for nuclear physicians.

The objective of this multicenter study is to provide a qualitative and consensual description of brain ${ }^{18} \mathrm{~F}$ FDG PET images in patients with suspected neurological long COVID. 


\section{Materials And Methods}

From the beginning of August 2021 to the end October 2021, brain ${ }^{18}$ F-FDG PET exams of patients referred for suspected neurological long COVID with a positive reverse transcription polymerase chain reaction (RT-PCR) and/or serology for the SARS-CoV-2 infection were retrospectively reviewed in three French Nuclear Medicine Departments (CHRU of Nancy, Timone Hospital, APHM Marseille and PitiéSalpêtrière Hospital, APHP, Paris). All patients responded to the current definition of long COVID by the World Health Organization (https://www.who.int/publications/i/item/WHO-2019-nCoV-Post_COVID19_condition-Clinical_case_definition-2021.1), with a PET evaluation performed at least 3 months after the demonstrated infection and while symptoms persisted.

The brain ${ }^{18} \mathrm{~F}$-FDG PET scan was recorded over a 10 to $15 \mathrm{~min}$ one bed acquisition, 30-45 min after injection of 2 to $3 \mathrm{MBq} / \mathrm{Kg}$ of ${ }^{18} \mathrm{~F}$-FDG. All subjects had fasted at least $6 \mathrm{~h}$ prior to receiving the injection and had blood glucose levels $<10 \mathrm{mmol} / \mathrm{L}$. All PET images were reconstructed with iterative OSEM methods, corrected for scatter, random and attenuation with a CT scan as usually performed in clinical routine for each department on three distinct cameras (Vereos, Philips ${ }^{\circledR}$ at Nancy; Biograph mCT Flow, Siemens ${ }^{\circledR}$ at Paris; Discovery 710, General Electric ${ }^{\circledR}$ at Marseille).

Experimented nuclear physicians from each department ( $A V, A K$ and $E G$ ) had to classify according to the same visual interpretation analysis brain ${ }^{18}$ F-FDG PET scans as being normal (or slightly non-specifically impaired), vs. mildly to moderate or severely affected within the previously reported long COVID hypometabolic pattern [4]. This typical hypometabolic long COVID pattern involves the fronto-orbital olfactory and limbic/paralimbic regions, the brainstem and cerebellum (4). A mild to moderate impairment was defined as incomplete hypometabolic pattern (two or three regions affected) or moderate hypometabolism affecting the 4 regions and was otherwise classified as a severe impairment. For this visual analysis, brain ${ }^{18} \mathrm{~F}$-FDG PET images were reoriented to the AC-PC axis, displayed through the French color scale by targeting the brain voxel with maximal uptake for normalization in intensity.

Images of control subjects, having performed brain ${ }^{18} \mathrm{~F}$-FDG PET scan with similar procedures of acquisition and reconstruction to patients for each center and corresponding to previously constituted database were also provided (NCT03345290, NCT02590276 and NCT00484523, respectively for Nancy, Paris and Marseille).

\section{Results}

Within the predefined period of 3 months, 10, 29 and 104 patients were addressed to the Nuclear Medicine departments for suspected neurological long COVID respectively in Nancy, Paris and Marseille. Based on the visual analysis, $53 \%$ of scans were interpreted as normal, $31 \%$ as mildly to moderate and $16 \%$ as severely affected according to the COVID hypometabolic pattern. Table 1 details results of this visual analysis per center. Importantly, this hypometabolic long COVID pattern was found identically in the three Nuclear Medicine Departments. 
Table 1

Results of the visual analysis among centers

\begin{tabular}{|llll|}
\hline & $\begin{array}{l}\text { Nancy } \\
(\mathbf{n = 1 0})\end{array}$ & $\begin{array}{l}\text { Paris } \\
(\mathbf{n = 2 9})\end{array}$ & $\begin{array}{l}\text { Marseille } \\
(\mathbf{n}=104)\end{array}$ \\
\hline Age (years-old) & $40.3 \pm 8.9$ & $47.3 \pm 12.4$ & $48.1 \pm 14.2$ \\
\hline Women & $5(50 \%)$ & $26(90 \%)$ & $67(64 \%)$ \\
\hline Time from symptoms onset (months) & $11.9 \pm 4.0$ & $14.4 \pm 4.9$ & $9.9 \pm 4.5$ \\
\hline Visual interpretation & & & \\
\hline Normal & $6(60 \%)$ & $14(48 \%)$ & $56(54 \%)$ \\
\hline Mild to moderate & $2(20 \%)$ & $11(38 \%)$ & $31(30 \%)$ \\
\hline Severe & $2(20 \%)$ & $4(14 \%)$ & $17(16 \%)$ \\
\hline
\end{tabular}

Illustrative examples of normal, mildly to moderate, or severely long COVID classified cases from the three nuclear medicine departments are depicted in Figure 1, with respectively no involvement, incomplete or moderate involvement, and severe involvement of the olfactory bulbs within the fronto-basal region and connected cerebral regions including limbic/paralimbic regions, the brainstem and the cerebellum. These cases are provided to help nuclear physicians to interpret brain ${ }^{18}$ F-FDG PET scans in case of suspected long COVID.

\section{Discussion}

This qualitative description provides arguments for a multicenter replication of the hypometabolic long COVID pattern previously reported at a single center level [4]. This pattern can be found at an individual level and help to objective metabolic abnormalities in patients with suspected neurological long COVID. It strengthens the fact that nuclear neurology can be helpful in routine for the evaluation of the long COVID [5].

The previously reported hypometabolic pattern is not visually found in all patients with suspicion of neurological long COVID. According to this retrospective review in a predefined period of inclusion, the mildly to moderate and severely hypometabolic long COVID patterns represent $47 \%$ of all patients addressed for this condition. This could explain why this hypometabolic pattern is not found in a series of 14 patients recently published in long COVID [6]. Possible explanations are discrepancy between visual and quantitative analyses, recovery from initial brain impairment (in mean, PET scans being performed at 10 months from symptoms onset) or fluctuations of brain abnormalities related to fluctuant clinical symptoms. Moreover, long COVID is a multisystemic disease, with also respiratory or cardiac symptoms that are persistent or fluctuating [1], which could also explain why some patients experiencing long COVID could have symptoms but without brain impairment. We also cannot formally rule out symptoms related to other extra-cerebral diseases than long COVID. 
Of note, regions affected within the previously reported pattern are known to be related to typical symptoms presented by patients with long COVID [7], especially olfactory loss, emotion disturbances, memory impairment, motor disorder, impaired balance and dysfunction of autonomous behaviors. Further studies are required to demonstrate such relationships between clinical characteristics and brain PET abnormalities at individual level.

This proposed pattern is easily applicable at visual interpretation, with consideration required for frontobasal paramedian regions, the brainstem and the cerebellum which are not classically as hypometabolic regions reported in other usual disorders. Even if not used for the current study, additional automatic semi-quantitative analysis with dedicated software could help to better identify subtle metabolic changes. Further larger studies should evaluate the benefit of adding such automatic analyses in the diagnosis of long COVID.

In any case, this pattern is clearly different from age-matched healthy controls and from those of neurodegenerative and psychiatric diseases $[8,9]$, and should be used in clinical routine by nuclear physicians to objective PET abnormalities related to the brain impairment in neurological long COVID.

\section{Declarations}

\section{Funding}

None

\section{Conflicts of interest/Competing interests}

The authors disclose no potential conflicts of interest related to the present work.

\section{Ethics approval and Consent to participate}

The retrospective observations required no ethical approval requirement other than informed consent. Informed consent was obtained from all individual participants included in the study.

\section{Consent for publication}

Not applicable

\section{Availability of data and material}

The data that support the findings of this study are available on request from the corresponding author (EG)

\section{Code availability}

Not applicable 


\section{Authors' contributions}

All authors contributed significantly to the analysis and interpretation of the data (AV, AK, EG), to the writing of the manuscript ( $A V, E G$ ) and to the revision of the manuscript (AK, PD, FG, DS, EG).

\section{References}

1. Greenhalgh T, Knight M, A'Court C, Buxton M, Husain L. Management of post-acute covid-19 in primary care. BMJ. 2020;370:m3026.

2. Douaud G, Lee S, Alfaro-Almagro F, Arthofer C, Wang C, McCarthy P, et al. Brain imaging before and after COVID-19 in UK Biobank [Internet]. Neurology; 2021 Jun. Available from: http://medrxiv.org/lookup/doi/10.1101/2021.06.11.21258690

3. Manca R, De Marco M, Ince PG, Venneri A. Heterogeneity in Regional Damage Detected by Neuroimaging and Neuropathological Studies in Older Adults With COVID-19: A CognitiveNeuroscience Systematic Review to Inform the Long-Term Impact of the Virus on Neurocognitive Trajectories. Front Aging Neurosci. 2021;13:646908.

4. Guedj E, Campion JY, Dudouet P, Kaphan E, Bregeon F, Tissot-Dupont $\mathrm{H}$, et al. 18F-FDG brain PET hypometabolism in patients with long COVID. Eur J Nucl Med Mol Imaging. 2021;48:2823-33.

5. Morbelli S, Ekmekcioglu O, Barthel H, Albert NL, Boellaard R, Cecchin D, et al. COVID-19 and the brain: impact on nuclear medicine in neurology. Eur J Nucl Med Mol Imaging. 2020;47:2487-92.

6. Dressing A, Bormann T, Blazhenets G, Schroeter N, Walter LI, Thurow J, et al. Neuropsychological profiles and cerebral glucose metabolism in neurocognitive Long COVID-syndrome. J Nucl Med. 2021;jnumed.121.262677.

7. Guedj E, Lazarini F, Morbelli S, Ceccaldi M, Hautefort C, Kas A, et al. Long COVID and the brain network of Proust's madeleine: targeting the olfactory pathway. Clin Microbiol Infect Off Publ Eur Soc Clin Microbiol Infect Dis. 2021;27:1196-8.

8. Guedj E, Tastevin M, Verger A, Richieri R. Brain PET imaging in psychiatric disorders. Ref Module Biomed Sci [Internet]. Elsevier; 2021 [cited 2021 Dec 10]. p. B9780128229606000000. Available from: https://linkinghub.elsevier.com/retrieve/pii/B9780128229606000909

9. Guedj E, Varrone A, Boellaard R, Albert NL, Barthel H, van Berckel B, et al. EANM procedure guidelines for brain PET imaging using [18F]FDG, version 3. Eur J Nucl Med Mol Imaging [Internet]. 2021 [cited 2021 Dec 10]; Available from: https://link.springer.com/10.1007/s00259-021-05603-w

\section{Figures}

\section{Figure 1}


Typical examples of brain ${ }^{18}$ F-FDG PET images of patients addressed for suspected long COVID in the three French Nuclear Medicine Departments, all presenting positive RT-PCR tests and/or serologies (cases from Nancy in the upper part, Paris in the middle part and Marseille in the lower part of the Figure). Cases in $\mathrm{A}$ are brain ${ }^{18} \mathrm{~F}$-FDG PET images identified as normal by the nuclear physician experts (a 44 years-old woman addressed 301 days from the COVID infection for persistence of dyspnea on exertion and tachycardia in the upper part, a 35 years-old woman addressed 113 days after an acute COVID for persistent asthenia, headache, sleep disturbance, polyarthralgia and memory/concentration impairment in the middle part, and a 50-years-old woman with memory complaints, headaches, and anosmia 314 days after the infection in the lower part), in B, patients classified as presenting mild to moderate long COVID hypometabolic pattern (a 41 years-old man who presented asthenia, dyspnea on exertion and cognitive disorders 315 days after the COVID acute affection in the upper part, a 52 years-old woman referred 342 days after COVID infection for cognitive complaint with language and memory difficulties, asthenia, insomnia and muscular weakness in the middle part, and a 55-years-old woman presenting memory complaints, loss of words, headaches, and dyspnea 168 days after the acute stage of the infection in the lower part) and in C, patients classified as presenting severe long COVID hypometabolic pattern (a 37 years-old-woman presenting cognitive impairment, dyspnea, orthostatic hypotension, hypersomnia and fever at 253 days from symptoms onset in the upper part, a 38 years-old-woman referred to brain ${ }^{18}$ F-FDG PET 545 days after an acute COVID with dysexecutive, memory difficulties, dizziness, limb paresthesia, asthenia, myalgia and polyarthralgia in the middle part, and a 20 years-oldwoman with persistence of functional symptoms with asthenia, headaches and memory/concentration impairment 170 days after the infection in the lower part). In D, brain ${ }^{18}$ F-FDG PET images of control cases in each Nuclear Medicine Departments (a 44 years-old -in the upper part, a 49 years-old-man in the middle part and a 22 years-old-woman in the lower part). 